\title{
Systematic Review Comparing the Effectiveness of Robotic verse Laparoscopic Liver Surgery in Colorectal Liver Metastasis (CRLM)
}

\author{
Nabeel Merali ${ }^{1, *}$, Hajra Ashraf ${ }^{2}$, Tarak Chouari ${ }^{2}$, Badriya Al Araimi ${ }^{3}$, Rajiv Lahiri ${ }^{1}$, Timothy A. Rockall ${ }^{1}$ \\ and Adam E. Frampton ${ }^{1}$ \\ 1 The Minimal Access Therapy Training Unit, University of Surrey, Daphne Jackson Road, Guildford, \\ Surrey GU2 7XH, UK; rajiv.lahiri@nhs.net (R.L.); t.rockall@nhs.net (T.A.R.); aframpton@nhs.net (A.E.F.) \\ 2 Croydon University Hospital, London CR7 7YE, UK; ashrafhajra@gmail.com (H.A.); t.chouari@nhs.net (T.C.) \\ 3 Armed Forces Hospital, Muscat 112, Oman; badria77@gmail.com \\ * Correspondence: nabeel.merali@gmail.com
}

check for

updates

Citation: Merali, N.; Ashraf, H.; Chouari, T.; Araimi, B.A.; Lahiri, R.; Rockall, T.A.; Frampton, A.E. Systematic Review Comparing the Effectiveness of Robotic verse Laparoscopic Liver Surgery in Colorectal Liver Metastasis (CRLM). Surgeries 2021, 2, 357-370. https:// doi.org/10.3390/surgeries2040035

Academic Editor: Matteo Donadon

Received: 31 August 2021

Accepted: 8 October 2021

Published: 12 October 2021

Publisher's Note: MDPI stays neutral with regard to jurisdictional claims in published maps and institutional affiliations.

Copyright: (c) 2021 by the authors. Licensee MDPI, Basel, Switzerland. This article is an open access article distributed under the terms and conditions of the Creative Commons Attribution (CC BY) license (https:/ / creativecommons.org/licenses/by/ $4.0 /)$.

\begin{abstract}
Introduction: Colorectal cancer (CRC) is the third most common cancer in the world. The liver is the most common site of metastasis with 15 to $25 \%$ of patients presenting with synchronous colorectal liver metastasis (CRLM). This study is aimed at evaluating the long- and short-term outcomes of laparoscopic and robotic CRLM surgery, and directly comparing their respective effectiveness. Methodology: A literature search was performed and all studies that reported on operative characteristics, oncological outcomes for CRLM, morbidity or mortality and cost-effectiveness on robotic or laparoscopic surgery were included. The study design was in keeping with the PRISMA guidelines. Results: From the initial 606 manuscripts identified, 19 studies were included in the final qualitative analysis. A total of 1340 patients with 1194 LLR (Laparoscopic Liver Resection) and 146 RLR (Robotic Liver Resection) cases were analysed. Within the LLR group, the average tumour size excised was $32.1 \mathrm{~mm}$ compared to the RLR group of $33.8 \mathrm{~mm}$. The average operative time in the LLR was $193 \mathrm{~min}, \mathrm{CI}$ of $95 \%$ (147.4 $\mathrm{min}$ to $238.6 \mathrm{~min}$ ) compared to RLR $257 \mathrm{~min}$, CI of $95 \%$ (201.5 $\mathrm{min}$ to $313.8 \mathrm{~min}$ ) with a $p$-value $<0.0001$. Estimated blood loss was lower in the RLR group (210 mL) compared with the LLR group $(246 \mathrm{~mL})$. Conclusion: Despite the higher operative cost, RLRs do not result in statistically better treatment outcomes, with the exception of lower estimated blood loss and excision of larger CRLMs. Operative time and total complication rate are significantly more favourable with LLRs. Our study has shown that robotic liver surgery is safe and feasible in well-selected patients.
\end{abstract}

Keywords: colorectal cancer; liver metastasis; oncology; surgery and education

\section{Introduction}

Colorectal cancer (CRC) is the third most common cancer worldwide [1]. According to Cancer Research UK, bowel cancer is the 4th most common cancer in the UK, accounting for $12 \%$ of all new cancer cases. There are around 42,000 new bowel cancer diagnoses in the UK every year, and it is responsible for approximately 8900 deaths annually [2]. The prognosis of colorectal cancer patients is in a large part determined by the presence of metastases. The liver is usually the first site of metastatic disease and may be the only site in $30-40 \%$ of patients with advanced disease [3]. Median survival without treatment is $<1$ year after disease presentation, and with a 5-year survival rate of $13 \%$ or less [4]. Safe and effective surgical procedures for treating colorectal cancer patients with liver metastases (CRCLM) have shifted from palliation to prolongation of survival and cure. Surgery is the only treatment that offers the prospect of a cure for CRLMs. In 1993, Woisetschläger R. performed the first laparoscopic CRLM [5]. Over the past decade, there is increasing evidence that laparoscopic liver resection (LLR) has been shown to be feasible and safe $[5,6]$. 
Benefits include reduced post-operative pain and ileus, shorter length of stay, rapid return to pre-operative activity and better cosmetic results. The criteria now used for measuring resect-ability are based on whether a macroscopically and microscopically complete (R0) resection of the liver can be achieved, and whether the volume of the liver remaining after resection will be adequate. Figure 1 below outlines the current $\mathrm{R}$ classification system nationally used, outlined by the United Kingdom Royal College of Pathologists.

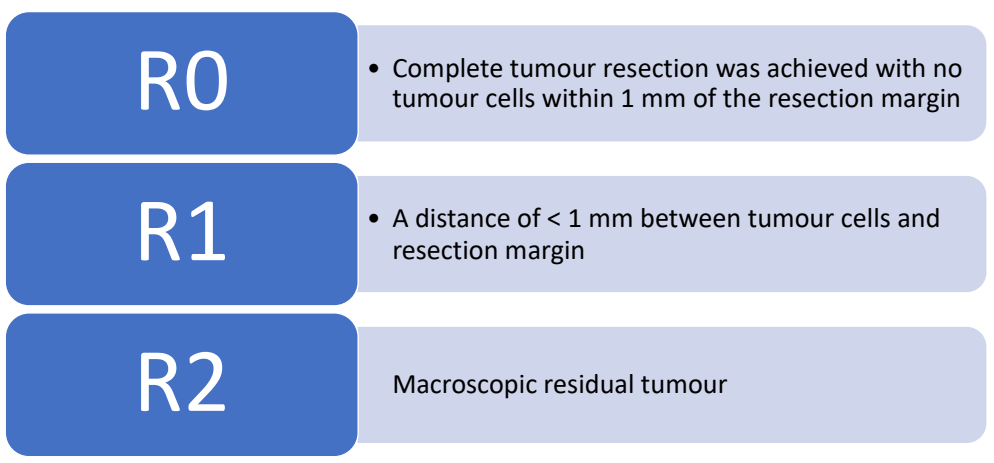

Figure 1. The United Kingdom Royal College of Pathologists has stated the current R classification [7].

The emergence of minimally invasive surgery for liver resection has flourished with the introduction of inventive technologies such as fibre optic imaging and ultrasonic haemostatic agents. In addition, growing evidence demonstrates that oncologic results are similar to open surgery in terms of R0 and R1 resections and overall 5-year survival. Operative results and postoperative variables were analysed by minor and major hepatectomy (e.g., more than two segments, right hepatectomy, or left hepatectomy) where appropriate. This study aimed to evaluate the short and long-term outcomes and directly compare the effectiveness of laparoscopic and robotic CRLM surgery.

\section{Materials and Methods}

The present study was designed according to the Preferred Reporting Items for Systematic Reviews and Meta-Analysis (PRISMA) guidelines. An electronic literature search of the databases (PubMed/MEDLINE, EMBASE and Cochrane database) in the English language was conducted in February 2020.

The following search terms were used:

- $\quad$ Robotic Surgery AND ((Colorectal Liver Metastasis OR (CRLM)) -25

- $\quad$ Laparoscopic surgery AND ((Colorectal Liver Metastasis OR (CRLM)) - 269

- Liver resection AND ((Colorectal Liver Metastasis OR (CRLM)) AND Robotic Surgery-21

- $\quad$ Liver resection AND ((Colorectal Liver Metastasis OR (CRLM)) AND Laparoscopic surgery-249

- ((COLORECTAL LIVER METASTASIS) OR (CRLM)) AND (("robotic liver surgery" OR ("liver surgery") OR (ROBOT ASSISTED SURGERY) OR (LAPAROSCOPIC SURGERY)) AND ((COST EFFECTIVENESS ANALYSIS) OR (cost effective))—26

Study Design: All titles and abstracts were screened for review with careful examination of the data to remove double counting of patients between series. A total of 19 articles were transferred to Mendeley` reference manager (Elsevier Inc., New York, USA) for full-text review based on the following inclusion criteria.

- That all studies were appropriate prospective and retrospective studies specifically addressing outcomes of patients with CRLM who underwent either laparoscopic or robotics resection.

- Studies that reported on perioperative characteristics, at least one postoperative outcome and oncological outcomes for CRLM, morbidity or mortality and costeffectiveness on robotic or laparoscopic surgery were included. 
The exclusion criteria included studies in which it was impossible to extract data specifically related to CRLM, review articles, editorials, case reports and letters. As well as nonhuman studies and those articles not translated into the English language.

Data Extraction and Statistical Analysis: Two reviewers conducted the literature search and data extraction. Any disagreement was resolved by consensus. Once the search was complete and the studies filtered, data were extracted from each study and analysed into a spreadsheet. Studies were then assessed for common themes which linked them together allowing for the identification of subgroups to allow for systematic review. We excluded results with insufficient data and reports with obvious errors. The PRISMA Flow diagram schematically depicts article selection (Figure 2).

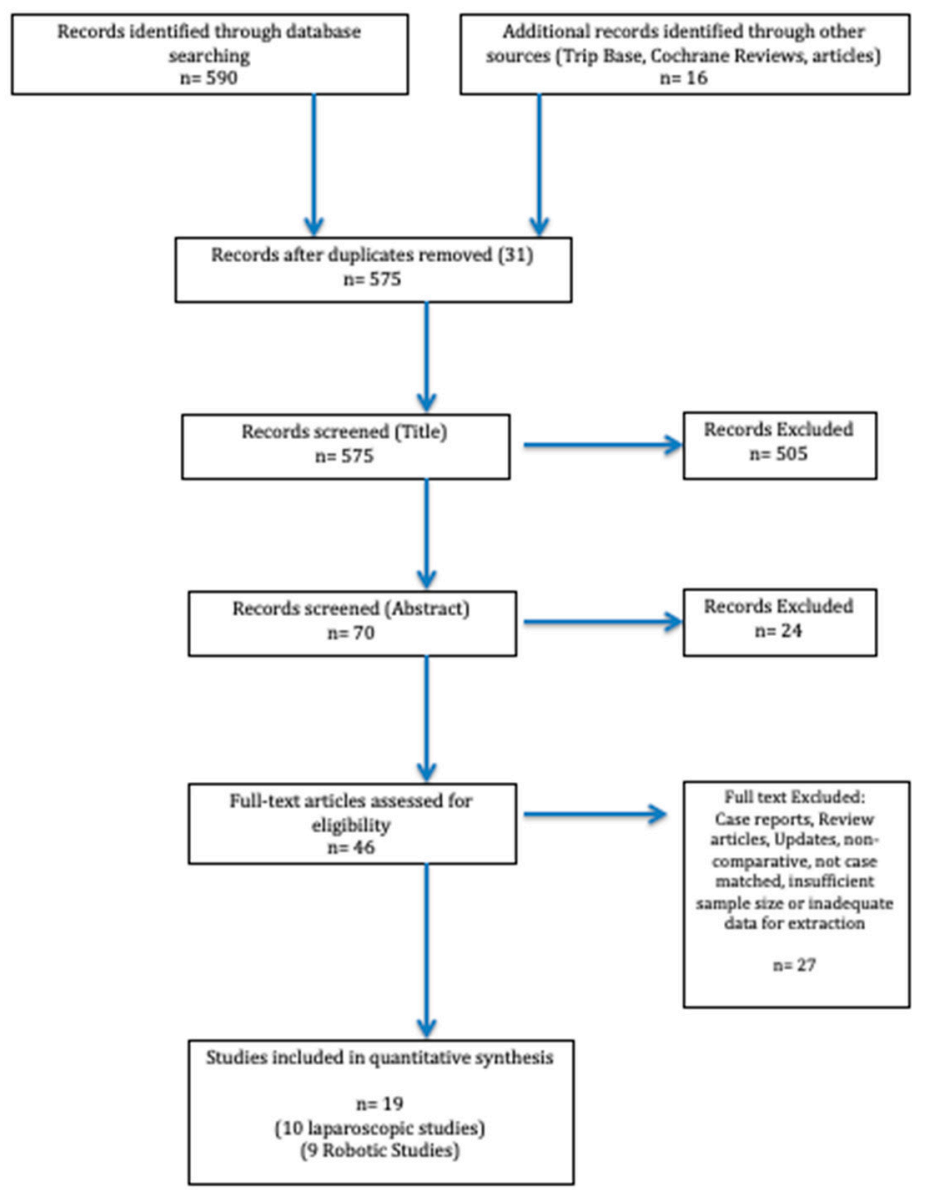

Figure 2. PRISMA flow diagram illustrating the article selection process for the systematic review. PRISMA, Preferred Reporting Items for Systematic Reviews and Meta-Analysis.

\section{Results}

The PubMed/MEDLINE, EMBASE and Cochrane database search identified a total of 606 studies. After excluding duplicates and following the PRISMA Guidelines, two independent reviewers carefully reviewed 70 abstracts. A total of 46 studies were identified that were meticulously appraised. In total, 28 articles were excluded according to the inclusion criteria, leaving 19 studies (10 laparoscopic, 9 robotic) that were included in our analysis. A total of 1340 patients with 1194 LLR (Laparoscopic Liver Resection) and 146 RLR (Robotic Liver Resection) cases were analysed. An overview of the reviewed articles and their respective intervention, management strategies and outcomes are highlighted in Tables 1 and 2 . 
Table 1. Outcomes of the Laparoscopic Liver Resections for CRLM.

\begin{tabular}{|c|c|c|c|c|c|c|c|c|c|c|}
\hline Article & Yue et al., 2018 & J. Shim et al., 2018 & $\begin{array}{l}\text { A. Fretland et al., } \\
2018\end{array}$ & $\begin{array}{l}\text { T. Nomi et al., } \\
2016\end{array}$ & $\begin{array}{l}\text { S. Ferretii et al., } \\
2015\end{array}$ & $\begin{array}{c}\text { R. Montalti et al., } \\
2014\end{array}$ & $\begin{array}{l}\text { S. Schiffman } \\
\text { et al., } 2014\end{array}$ & $\begin{array}{l}\text { B. Topal et. al. } \\
\quad 2012\end{array}$ & $\begin{array}{l}\text { H. Topal et al., } \\
2012\end{array}$ & A. Hilal 2012 \\
\hline Type of study & Retrospective & Retrospective & $\begin{array}{c}\text { Randomised } \\
\text { control trial }\end{array}$ & Retrospective & Retrospective & Retrospective & $\begin{array}{l}\text { Retrospective, } \\
\text { case series }\end{array}$ & Retrospective & Prospective study & $\begin{array}{l}\text { retrospective, } \\
\text { single centre }\end{array}$ \\
\hline No. of Cases & 241 & 22 & 129 & 120 & 142 & 114 & 242 & 81 & 20 & 83 \\
\hline Age (years) & $\begin{array}{c}67(60-69) \& \\
74 \text { (70-78) NB: } \\
\text { Two Age Groups }\end{array}$ & 65.6 & 67 & 61 & 66 & 66.4 & 60.8 & 64.3 & 57.6 & $66(32-85)$ \\
\hline $\begin{array}{c}\text { Preoperative } \\
\text { systemic } \\
\text { chemotherapy }\end{array}$ & 187 & 21 & 77 & 71 & 25 & 78 & 128 & 55 & 15 & Not mentioned \\
\hline $\begin{array}{l}\text { Type of liver } \\
\text { resection }\end{array}$ & $\begin{array}{c}18 \text { Major } \\
\text { (Left lateral } \\
\text { sectionectomy), } \\
\text { 223 Minor (wedge/ } \\
\text { sectionectomy) }\end{array}$ & 2 Major, 20 Minor & Not mentioned & 83 Major cases & $\begin{array}{l}39 \text { Major cases, } \\
103 \text { Minor cases }\end{array}$ & $\begin{array}{l}8 \text { major cases, } \\
\text { Postero-superior } \\
\text { resections } 52 \\
(45.6 \%)\end{array}$ & $\begin{array}{l}104 \text { major cases, } \\
133 \text { minor cases }\end{array}$ & 18 Major, 34 Minor & 20 Major & Major 27, Minor 56 \\
\hline Tumour Size & $\begin{array}{c}30 \mathrm{~mm}(10-50) \& \\
20 \mathrm{~mm}(10-40)\end{array}$ & $16 \mathrm{~mm}(6-101)$ & Not mentioned & 33 mm (5-170) & $28 \mathrm{~mm}(2-100)$ & $40.8 \mathrm{~mm} \pm 2.9$ & $31 \mathrm{~mm}$ & $>50 \mathrm{~mm}-7$ cases & 40 mm (4-70) & 25 mm (5-105) \\
\hline No. of CRLM & $2(1-4)$ & $1(1-2)$ & 1.5 & $2(1-8)$ & $1(1-9)$ & 1.7 & 1.37 & 2 & $2(1-6)$ & $\begin{array}{l}1 \text { lesion ( } 54 \text { cases), } \\
2 \text { lesions ( } 21 \text { cases), } \\
>3 \text { lesions ( } 8 \text { cases) }\end{array}$ \\
\hline CRLM & Not mentioned & $\begin{array}{l}14 \text { synchronous, } \\
8 \text { Metachronous }\end{array}$ & 77 Synchronous & 67 synchronous & 142 synchronous & 54 synchronous & Not mentioned & $\begin{array}{l}\text { Metachronous 45, } \\
\text { synchronous } 36\end{array}$ & $\begin{array}{l}3 \text { synchronous } \\
\text { resection }\end{array}$ & not mentioned \\
\hline $\begin{array}{c}\text { Duration of } \\
\text { surgery (min) }\end{array}$ & $\begin{array}{c}190(150-290) \& \\
180(160-260)\end{array}$ & $135(40-360)$ & $123(108-138)$ & $245(60-540)$ & $120(15-600)$ & $276 \pm 10.1$ & 248.7 & $120(80-200)$ & $257.5(75-360)$ & $220 \min (40-540)$ \\
\hline $\begin{array}{l}\text { conversion rate } \\
\text { (lap to open) }\end{array}$ & 8 cases & Not mentioned & 9 cases & 8 cases $(6.7 \%)$ & 7 cases & 17 cases & Not mentioned & 6 cases & Not mentioned & 5 cases \\
\hline $\begin{array}{l}\text { estimated blood } \\
\text { loss median } \\
\text { (range) }\end{array}$ & $\begin{array}{l}240(160-410) \& \\
260(180-430)\end{array}$ & $100(30-950)$ & $300(224-375)$ & $200(0-3000)$ & $200(0-1800)$ & $250(0-2800)$ & $262.5 \mathrm{~mL}$ & $50(10-300)$ & $550(100-4000)$ & $300(20-3000)$ \\
\hline $\begin{array}{c}\text { Pringle } \\
\text { maneuver use }\end{array}$ & Not mentioned & 22 cases & Not mentioned & $\begin{array}{l}\text { Yes performed, } \\
\text { pringle time } \\
\text { median range: } \\
25(8-75)\end{array}$ & 17 cases & $12(10.5 \%)$ & Not mentioned & Not mentioned & 4 cases & $\begin{array}{l}\text { Yes performed for } \\
\text { all cases }\end{array}$ \\
\hline $\begin{array}{l}\text { Resection margin } \\
\text { size, R1, etc. }\end{array}$ & $\begin{array}{c}(\mathrm{R} 0=241 / \mathrm{R} 1=0 / \\
\mathrm{R} 2=0)\end{array}$ & Not mentioned & $\begin{array}{c}<1 \mathrm{~mm} \text { ( } 29 \text { cases), } \\
>1 \mathrm{~mm}(92 \text { cases), } \\
\text { involved ( } 8 \text { cases), } \\
\text { missed ( } 4 \text { cases) }\end{array}$ & $\begin{array}{c}R 0 \text { resection }= \\
113 \text { cases }(94.2 \%)\end{array}$ & $\begin{array}{c}\text { postive surgical } \\
\text { margins }=10 \text { cases }\end{array}$ & $\begin{array}{c}\mathrm{R} 1<1 \mathrm{~mm}= \\
14 \text { cases, } \\
\mathrm{R} 0=100 \text { cases }\end{array}$ & $\begin{array}{c}\text { R1 Margin } \\
\text { positivity } 5.5 \%= \\
12 \text { Cases }\end{array}$ & Not mentioned & $\begin{array}{c}\text { R1-1 Case, R0 = } \\
19 \text { Cases with } \\
\text { median tumour } \\
\text { free margin of } \\
7.5 \mathrm{~mm}(0-20)\end{array}$ & $\begin{array}{c}\text { R0-80 Cases } \\
(97 \%) \text { R1-3 cases }\end{array}$ \\
\hline $\begin{array}{l}\text { RO resection \% } \\
\text { per cases (LLR) }\end{array}$ & $240 / 240$ & $\mathrm{~N} / \mathrm{A}$ & $121 / 129$ & $113 / 120$ & $132 / 142$ & $100 / 114$ & $230 / 242$ & $\mathrm{~N} / \mathrm{A}$ & $19 / 20$ & $80 / 83$ \\
\hline
\end{tabular}


Table 1. Cont.

\begin{tabular}{|c|c|c|c|c|c|c|c|c|c|c|}
\hline Article & Yue et al., 2018 & J. Shim et al., 2018 & $\begin{array}{l}\text { A. Fretland et al., } \\
2018\end{array}$ & $\begin{array}{l}\text { T. Nomi et al., } \\
2016\end{array}$ & $\begin{array}{l}\text { S. Ferretii et al., } \\
2015\end{array}$ & $\begin{array}{l}\text { R. Montalti et al., } \\
2014\end{array}$ & $\begin{array}{l}\text { S. Schiffman } \\
\text { et al., } 2014\end{array}$ & $\begin{array}{l}\text { B. Topal et. al. } \\
2012\end{array}$ & $\begin{array}{l}\text { H. Topal et al., } \\
2012\end{array}$ & A. Hilal 2012 \\
\hline $\begin{array}{l}\text { Length of hospital } \\
\text { stay }\end{array}$ & $\begin{array}{c}9(7-23) \& 10 \\
(7-32)\end{array}$ & $8.5(5-22)$ & 2.3 days $(1.8-3)$ & $7(4-42)$ & $8(3-84)$ & 6.0 days \pm 0.28 & 6.5 days & 5 days $(3-7)$ & $8(5-51)$ & $5(2-12)$ \\
\hline $\begin{array}{l}\text { readmission rate } \\
\text { within } 30 \text { days }\end{array}$ & Not Mentioned & 0 & 13 cases & Not Mentioned & Not Mentioned & Not Mentioned & Not Mentioned & 0 & 0 cases & 0 \\
\hline $\begin{array}{l}\text { Re admission } \\
\text { Operation rate } \\
\text { within } 30 \text { days }\end{array}$ & Not Mentioned & 0 & 5 cases & Not Mentioned & 11 & 6 cases & Not Mentioned & 0 & 0 cases & $\begin{array}{c}\text { Not specifically } \\
\text { mentioned for } \\
\text { CRLMs, however } \\
5 \text { cases returned to } \\
\text { theatre }\end{array}$ \\
\hline Complication \% & 30 & 9 & 18 & 41 & 31 & 20 & 20 & 14 & 35 & 11 \\
\hline $\begin{array}{c}\text { No. of } \\
\text { Complications }\end{array}$ & $\begin{array}{l}73 \text { cases, Not } \\
\text { specified }\end{array}$ & 2 cases & 24 cases & $\begin{array}{c}50 \text { cases (39 liver } \\
\text { specific), overall } \\
21 \text { of } 50 \text { cases were } \\
\text { major } \\
\text { complications }\end{array}$ & 44 cases, & 23 cases & $\begin{array}{l}48 \text { cases }(12.8 \% \\
\text { Liver specific) }\end{array}$ & 11 cases & 7 cases & $\begin{array}{c}9 \text { major } \\
\text { complications } \\
(11 \%)\end{array}$ \\
\hline $\begin{array}{c}\text { Type of } \\
\text { complication }\end{array}$ & & & & & & & & & & major \\
\hline $\begin{array}{l}\text { Clavien-Dindo } \\
\text { system } \\
\text { Grade (G1-5) }\end{array}$ & Not mentioned & Grade 1 ( 2 cases) & Not mentioned & $\mathrm{G} 5=1$ & $\begin{array}{c}\mathrm{G} 1=2, \mathrm{G} 2=14 \\
\mathrm{G} 3=20, \mathrm{G} 4=5 \\
\mathrm{G} 5=3\end{array}$ & Not mentioned & $\mathrm{G} 5=1$ & Not mentioned & Not mentioned & not mentioned \\
\hline Bleeding & $\mathrm{n} / \mathrm{a}$ & 0 & 4 & 2 & 3 & 10 & $\mathrm{n} / \mathrm{a}$ & 2 & 1 & 5 \\
\hline Liver abscess & $\mathrm{n} / \mathrm{a}$ & 0 & 2 & $\mathrm{n} / \mathrm{a}$ & 0 & $\mathrm{n} / \mathrm{a}$ & $\mathrm{n} / \mathrm{a}$ & 1 & 0 & 2 \\
\hline Biliary Leakage & $\mathrm{n} / \mathrm{a}$ & 0 & 1 & 20 & 6 & $\mathrm{n} / \mathrm{a}$ & $\mathrm{n} / \mathrm{a}$ & 0 & 0 & 2 \\
\hline Liver insufficiency & $\mathrm{n} / \mathrm{a}$ & 0 & 1 & 12 & 1 & $\mathrm{n} / \mathrm{a}$ & $\mathrm{n} / \mathrm{a}$ & 1 & 1 & 0 \\
\hline DVC Sepsis & $\mathrm{n} / \mathrm{a}$ & 0 & 0 & $\mathrm{n} / \mathrm{a}$ & 0 & $\mathrm{n} / \mathrm{a}$ & $\mathrm{n} / \mathrm{a}$ & 1 & 1 & 0 \\
\hline colorectal leaakge & $\mathrm{n} / \mathrm{a}$ & 1 & 0 & $\mathrm{n} / \mathrm{a}$ & 8 & $\mathrm{n} / \mathrm{a}$ & $\mathrm{n} / \mathrm{a}$ & 0 & 1 & 0 \\
\hline Pulmonary/Cardiac & $\mathrm{n} / \mathrm{a}$ & 0 & 6 & 4 & 8 & $\mathrm{n} / \mathrm{a}$ & $\mathrm{n} / \mathrm{a}$ & 0 & 1 & 0 \\
\hline MOF (Perionitis) & $\mathrm{n} / \mathrm{a}$ & 0 & 3 & 8 & 1 & $\mathrm{n} / \mathrm{a}$ & $\mathrm{n} / \mathrm{a}$ & 1 & 1 & 0 \\
\hline Death & $n / a$ & 0 & 0 & 1 & 3 & $\mathrm{n} / \mathrm{a}$ & 1 & 0 & 0 & 0 \\
\hline
\end{tabular}


Table 1. Cont.

\begin{tabular}{|c|c|c|c|c|c|c|c|c|c|c|}
\hline Article & Yue et al., 2018 & J. Shim et al., 2018 & $\begin{array}{l}\text { A. Fretland et al., } \\
\qquad 2018\end{array}$ & $\begin{array}{l}\text { T. Nomi et al., } \\
2016\end{array}$ & $\begin{array}{l}\text { S. Ferretii et al., } \\
2015\end{array}$ & $\begin{array}{l}\text { R. Montalti et al., } \\
2014\end{array}$ & $\begin{array}{l}\text { S. Schiffman } \\
\text { et al., } 2014\end{array}$ & $\begin{array}{l}\text { B. Topal et. al. } \\
\qquad 2012\end{array}$ & $\begin{array}{l}\text { H. Topal et al., } \\
2012\end{array}$ & A. Hilal 2012 \\
\hline $\begin{array}{l}\text { 90-days mortality } \\
\text { rate and Estimated } \\
\text { DFS/OS }\end{array}$ & $\begin{array}{c}2 \text { cases }=1 \text { case in } \\
\text { the elderly group } \\
\text { due to liver failure } \\
\text { and } 1 \text { case in } \\
\text { Middle age group } \\
\text { due to metastasis } \\
\text { to CNS. FU at } 34 \\
\text { months in elderly } \\
\text { group }=32 \text { deaths } \\
\text { (29 reoccurence of } \\
\text { disease), Middle } \\
\text { age group }=53 \\
\text { deaths }(48 \\
\text { reoccurence) }\end{array}$ & 0 & Not mentioned & $\begin{array}{c}\text { one case, study } \\
\text { showed that OS } \\
\text { and RFS were } \\
\text { acceptable in } \\
\text { patients with } \\
\text { CRLMs }(5 \mathrm{~cm}) \text {. } \\
\text { Furthermore, } \\
\text { multivariate } \\
\text { analysis did not } \\
\text { identify the tumor } \\
\text { size as a } \\
\text { prognostic factor }\end{array}$ & $\begin{array}{c}3 \text { deaths within } \\
30 \text { day mortalitiy } \\
\text { Median FU of } 29 \\
\text { (1-108 months), } \\
40 \text { patients } \\
\text { developed tumour } \\
\text { reoccurence }\end{array}$ & $\begin{array}{c}66(57.9 \%) \text { of the } \\
\text { cases reoccurence } \\
\text { of disease in } 30 \\
\text { month follow up } \\
\text { R1 margins were } \\
\text { significantly } \\
\text { related to lower } \\
\text { RFS survival } \\
(p=0.038) \text { but did } \\
\text { not affect OS. }\end{array}$ & $\begin{array}{c}1 \text { death-massive } \\
\text { bleed from portal } \\
\text { vein }\end{array}$ & Not mentioned & $\begin{array}{l}\text { The estimated DFS } \\
\text { and OS rates in } \\
\text { the LMLR group } \\
\text { at } 1,2, \text { and } 5 \text { years } \\
\text { were } 60,49 \text {, and } 43 \\
\text { and } 90,80 \text {, and } \\
48 \% \text { respectively }\end{array}$ & $\begin{array}{c}22 \text { months FU } \\
2 \text { Year DFS/OS } \\
64 \% / 80 \%\end{array}$ \\
\hline $\begin{array}{l}\text { Cost (might not be } \\
\text { directly stated) }\end{array}$ & Not Mentioned & $\begin{array}{c}\text { In conclusion, no } \\
\text { significant } \\
\text { differences in } \\
\text { postoperative } \\
\text { outcomes were } \\
\text { observed between } \\
\text { LSLR and OSLR } \\
\text { except length of } \\
\text { hospital stay, the } \\
\text { number of liver } \\
\text { metastasis, and } \\
\text { the resection } \\
\text { margin. }\end{array}$ & $\begin{array}{l}\text { cost difference } \\
\text { from open surgery } \\
\text { is } \$ 94,000\end{array}$ & $\begin{array}{l}\text { study suggests } \\
\text { that LLR for large } \\
\text { CRLMs can be } \\
\text { performed safely } \\
\text { with acceptable } \\
\text { long-term } \\
\text { outcomes in } \\
\text { selected patients. } \\
\text { Therefore, tumor } \\
\text { size of CRLM } \\
\text { should not } \\
\text { interfere when } \\
\text { selecting the } \\
\text { surgical approach }\end{array}$ & Not Mentioned & Not Mentioned & Not Mentioned & Not mentioned & Not Mentioned & $\begin{array}{c}\text { Overall, our data } \\
\text { including a large } \\
\text { proportion } \\
\text { of major } \\
\text { hepatectomies } \\
\text { confirm the } \\
\text { well-established } \\
\text { advantages of } \\
\text { laparoscopic liver } \\
\text { surgery in terms } \\
\text { of reduced } \\
\text { high-dependency } \\
\text { unit and } \\
\text { postoperative } \\
\text { length of stay. }\end{array}$ \\
\hline
\end{tabular}


Table 2. Outcomes of the Robotic Liver Resections for CRLM.

\begin{tabular}{|c|c|c|c|c|c|c|c|c|c|}
\hline Article & $\begin{array}{l}\text { R. Beard } \\
\text { et al.2020 }\end{array}$ & S. Guadagni et al., 2019 & $\begin{array}{l}\text { F. Guerra et al., } \\
2018\end{array}$ & $\begin{array}{l}\text { R. Dwyer et al., } \\
2018\end{array}$ & A. Tsung et al., 2014 & $\begin{array}{l}\text { Eric C.H. Lai et al., } \\
2011\end{array}$ & $\begin{array}{l}\text { P.C. Giulianotti } \\
\text { et al., } 2011\end{array}$ & L. Casciola et al., 2011 & E. Berber et al., 2010 \\
\hline Type of Study & $\begin{array}{l}\text { retrospective } \\
\text { multicentre }\end{array}$ & Retrospective & $\begin{array}{l}\text { retrospective } \\
\text { multicentre }\end{array}$ & $\begin{array}{l}\text { retrospective } \\
\text { single centre }\end{array}$ & $\begin{array}{l}\text { Retrospective } \\
\text { Single centre }\end{array}$ & Retrospective & $\begin{array}{c}\text { retrospective, } \\
2 \text { different centers } \\
\text { by a single surgeon. }\end{array}$ & $\begin{array}{l}\text { Prospective series } \\
\text { single study }\end{array}$ & $\begin{array}{l}\text { retrospective single } \\
\text { centre, } 9 \text { robotic cases } \\
\text { (4 for CRLM) }\end{array}$ \\
\hline No. of Patients & 115 & 20 robotic assisted & $\begin{array}{l}59 \text { (USS guided } \\
\text { Robotic) }\end{array}$ & 6 & 21 & 6 Robtic assisted & 16 & 14 Robotic assited LHR & 4 \\
\hline Age (years) & 61 & 66 & 64 & 59.3 & 58.4 & 68.2 & 57 & 66.4 & 68.6 \\
\hline $\begin{array}{l}\text { Preoperative sys- } \\
\text { temic chemother- } \\
\text { apy cases }\end{array}$ & $63(54.8 \%)$ & 12 & $14(17 \%)$ & 6 & Not mentioned & Not mentioned & not mentioned & not mentioned & not mentioned \\
\hline $\begin{array}{l}\text { Type of liver } \\
\text { resection }\end{array}$ & $\begin{array}{l}18 \text { Minor, } \\
97 \text { Major }\end{array}$ & 20 Major, (3 simultaneous) & 39 major, 43 minor & $\begin{array}{l}1 \text { Major, } 3 \text { Minor, } \\
1 \text { ablation } \\
\text { (large sege- } \\
\text { ment } 7 \text { near vas- } \\
\text { cular structures), } \\
1 \text { no identifi- } \\
\text { able lesion }\end{array}$ & 16 minor, 4 major & $\begin{array}{l}6 \text { Major ( } 3 \text { wedge } \\
\text { resections, } 3 \text { left lat- } \\
\text { eral sectionectomy) }\end{array}$ & $\begin{array}{c}4 \text { minor } \\
\text { ( }<3 \text { segments) and } \\
12 \text { major resections }\end{array}$ & $\begin{array}{l}14 \text { minor resections, } 11 \\
\text { simultaneous procedues }\end{array}$ & $\begin{array}{l}4 \text { minor (peripheral } \\
\text { segments) }\end{array}$ \\
\hline Tumour Size & $\begin{array}{c}25 \mathrm{~mm} \\
(3 \mathrm{~mm}-122 \mathrm{~mm})\end{array}$ & $30 \mathrm{~mm} \pm(18)$ & $27 \mathrm{~mm}(4-130)$ & Not mentioned & $\begin{array}{c}\text { Minor-31 mm } \\
(20-55), \\
\text { Major-37 mm } \\
(24.5-50 \mathrm{~mm})\end{array}$ & $33 \mathrm{~mm} \pm(16)$ & $\begin{array}{l}47 \mathrm{~mm} \text { (range, } \\
12-110)\end{array}$ & not mentioned & $32 \pm 13 \mathrm{~mm}$ \\
\hline CRLM & $\begin{array}{l}49 \text { synchronous } \\
\text { cases }\end{array}$ & 6 synchronous cases & 16 synchronous & $\begin{array}{l}6 \text { (Liver first } \\
\text { approach) }\end{array}$ & not mentioned & $\begin{array}{l}9 \text { metachronous, } \\
5 \text { synchronous }\end{array}$ & not mentioned & $\begin{array}{l}3 \text { metachronous, } \\
11 \text { synchronous }\end{array}$ & not mentioned \\
\hline $\begin{array}{l}\text { Duration of } \\
\text { surgery (min) }\end{array}$ & $272 \pm 115$ & $198.5 \pm(98)$ & $210 \min (50-600)$ & $401(349-506)$ & $253 \min (180-355)$ & $190.2 \pm 91.6$ & $\begin{array}{c}270 \text { min (range, } \\
90-660)\end{array}$ & $280 \pm 115 \mathrm{~min}$ & $258.5 \pm 27.9 \mathrm{~min}$ \\
\hline $\begin{array}{l}\text { Conversion rate } \\
\text { (robotic to open) }\end{array}$ & $6(5.2 \%)$ & 0 & 7 cases $(12 \%)$ & 0 & 4 cases & 0 & 4 cases & 1 case & 1 case \\
\hline $\begin{array}{l}\text { estimated blood } \\
\text { loss median } \\
\text { (range) }\end{array}$ & Not mentioned & $250(200-300)$ & 200ml (0-1500) & $316(150-1000)$ & $200(50-337.5)$ & $75(20-200)$ & $\begin{array}{l}262 \mathrm{~mL} \text { (range, } \\
20-2000 \text { ) }\end{array}$ & $245 \pm 254(0-1000)$ & $136 \pm 61 \mathrm{~mL}$ \\
\hline $\begin{array}{c}\text { Pringle } \\
\text { maneuver use }\end{array}$ & $\begin{array}{l}\text { Used at the } \\
\text { discretion of the } \\
\text { surgeon }\end{array}$ & Not mentioned & $\begin{array}{l}18 \text { cases }(30 \%) \\
\text { during } \\
\text { parenchymal } \\
\text { transection }\end{array}$ & Not mentioned & not mentioned & $\begin{array}{c}3 \text { cases, Mean time } \\
46.7 \mathrm{~min}\end{array}$ & 0 & $\begin{array}{l}12 \text { cases, mean clamp } \\
\quad \text { time } 68.9 \pm 31.7\end{array}$ & not mentioned \\
\hline
\end{tabular}


Table 2. Cont.

\begin{tabular}{|c|c|c|c|c|c|c|c|c|c|}
\hline Article & $\begin{array}{l}\text { R. Beard } \\
\text { et al.2020 }\end{array}$ & S. Guadagni et al., 2019 & $\begin{array}{c}\text { F. Guerra et al., } \\
2018\end{array}$ & $\begin{array}{c}\text { R. Dwyer et al., } \\
2018\end{array}$ & A. Tsung et al., 2014 & $\begin{array}{l}\text { Eric C.H. Lai et al., } \\
2011\end{array}$ & $\begin{array}{l}\text { P.C. Giulianotti } \\
\text { et al., } 2011\end{array}$ & L. Casciola et al., 2011 & E. Berber et al., 2010 \\
\hline $\begin{array}{l}\text { Resection margin } \\
\text { size, R1, etc. }\end{array}$ & $\begin{array}{l}\text { R0 }=84(73.7 \%), \\
\text { R1 }=19(16.7 \%)\end{array}$ & R0-20 cases $(100 \%)$ & $\begin{array}{l}\text { R0 resection- } \\
54 \text { cases }(92 \%) \\
\text { R1-5 cases }\end{array}$ & $\begin{array}{c}\mathrm{R} 0 \\
\text { resection-100\% }\end{array}$ & $\begin{array}{l}\text { R0 negative } \\
\text { margin-95\% } \\
\text { of cases }\end{array}$ & $\begin{array}{l}\text { RO- }-5 \text { cases, } \\
\text { R1-1 case }\end{array}$ & $\begin{array}{c}\text { mean surgical } \\
\text { resection margin } \\
25 \mathrm{~mm} \text { (range, } \\
5-70) .\end{array}$ & not mentioned & $\begin{array}{c}\text { resection margin } \\
11 \mathrm{~mm}, \\
\text { Resectionmargins wer } \\
\text { negative for a tumour } \\
\text { at the time of resection }\end{array}$ \\
\hline $\begin{array}{l}\text { No. of R0 } \\
\text { resection per cases }\end{array}$ & $84 / 115$ & $20 / 20$ & $27 / 29$ & $6 / 6$ & $20 / 21$ & $5 / 6$ & $\mathrm{n} / \mathrm{a}$ & $\mathrm{n} / \mathrm{a}$ & $4 / 4$ \\
\hline $\begin{array}{l}\text { Length of } \\
\text { hospital stay }\end{array}$ & $5(3-6)$ & $4.7 \pm 1.8$ day & $6.7 \pm 6.2$ days & $4.5(3-10)$ & $4.0(3.0-5.5)$ & 6.8 mean day \pm 2.8 & $\begin{array}{l}7 \text { days median } \\
\quad(2-26)\end{array}$ & $\begin{array}{l}6.8 \pm 2.8 \text { days, NB for } \\
\text { synchronous CRLM it } \\
\text { was } 9 \pm 2.6 \text { days }\end{array}$ & not mentioned \\
\hline $\begin{array}{l}\text { readmission rate } \\
\text { within } 30 \text { days }\end{array}$ & $8(7.0 \%)$ & 0 & Not mentioned & 0 & 0 & 0 & not mentioned & 0 & 0 \\
\hline $\begin{array}{l}\text { Re admission } \\
\text { Operation rate } \\
\text { within } 30 \text { days }\end{array}$ & $1(0.9 \%)$ & 0 & 1 & 0 & 1 & 1 & not mentioned & 1 & not mentioned \\
\hline Complication \% & 10.4 & 25 & 27 & 67 & 52 & 16 & not mentioned & 28 & 11 \\
\hline $\begin{array}{c}\text { No. of } \\
\text { Complications }\end{array}$ & 12 & 5 cases & 16 cases $(27 \%)$ & 4 Cases & 11 cases & 1 case & not mentioned & 4 cases & $\begin{array}{l}11 \% \text { of the robotic } \\
\text { (mixed result) }\end{array}$ \\
\hline $\begin{array}{c}\text { Type of } \\
\text { complication } \\
\text { (Claven-Dindo) } \\
\text { Grade (G1-5) }\end{array}$ & $\mathrm{G} 3=12$ cases & $\begin{array}{l}\text { G1- } 1 \text { case, } \\
\text { G2-4 cases }\end{array}$ & $\begin{array}{c}\text { G1-2 }(13 \text { cases, } \\
22 \%), \text { G3-4 } \\
(3 \text { cases } 5 \%)\end{array}$ & Not mentioned & G3-4-one case & G2 (1 case) & $\begin{array}{l}\text { unable to distinguish } \\
\text { complications from } \\
\text { other cases } \\
\text { (heterogenous data) }\end{array}$ & $\begin{array}{l}4 \text { cases, unable to } \\
\text { distinguish complications } \\
\text { from other cases } \\
\text { (heterogenous data) }\end{array}$ & $\begin{array}{l}\text { unable to distinguish } \\
\text { complications from } \\
\text { other cases } \\
\text { (heterogenous data) }\end{array}$ \\
\hline SSI & $\mathrm{n} / \mathrm{a}$ & $\mathrm{n} / \mathrm{a}$ & $\mathrm{n} / \mathrm{a}$ & 1 & $\mathrm{n} / \mathrm{a}$ & 0 & $\mathrm{n} / \mathrm{a}$ & $\mathrm{n} / \mathrm{a}$ & $\mathrm{n} / \mathrm{a}$ \\
\hline Bleeding & 3 & $\mathrm{n} / \mathrm{a}$ & 3 & 0 & $\mathrm{n} / \mathrm{a}$ & 0 & $\mathrm{n} / \mathrm{a}$ & 2 & 1 \\
\hline $\begin{array}{l}\text { Liver/Intraabdominal } \\
\text { abscess/collection }\end{array}$ & 7 & $\mathrm{n} / \mathrm{a}$ & $\mathrm{n} / \mathrm{a}$ & 2 pelvic & $\mathrm{n} / \mathrm{a}$ & 0 & $\mathrm{n} / \mathrm{a}$ & $\mathrm{n} / \mathrm{a}$ & $\mathrm{n} / \mathrm{a}$ \\
\hline Biliary Leakage & $\mathrm{n} / \mathrm{a}$ & $\mathrm{n} / \mathrm{a}$ & 1 & 0 & $\mathrm{n} / \mathrm{a}$ & 1 & $\mathrm{n} / \mathrm{a}$ & 1 & $\mathrm{n} / \mathrm{a}$ \\
\hline Liver insufficiency & $\mathrm{n} / \mathrm{a}$ & $\mathrm{n} / \mathrm{a}$ & $\mathrm{n} / \mathrm{a}$ & 0 & $\mathrm{n} / \mathrm{a}$ & 0 & $\mathrm{n} / \mathrm{a}$ & $\mathrm{n} / \mathrm{a}$ & $\mathrm{n} / \mathrm{a}$ \\
\hline DVC Sepsis & $\mathrm{n} / \mathrm{a}$ & $\mathrm{n} / \mathrm{a}$ & $\mathrm{n} / \mathrm{a}$ & 0 & $\mathrm{n} / \mathrm{a}$ & 0 & $\mathrm{n} / \mathrm{a}$ & $\mathrm{n} / \mathrm{a}$ & $\mathrm{n} / \mathrm{a}$ \\
\hline colorectal leaakge & $\mathrm{n} / \mathrm{a}$ & $\mathrm{n} / \mathrm{a}$ & $\mathrm{n} / \mathrm{a}$ & 1 & $\mathrm{n} / \mathrm{a}$ & 0 & $\mathrm{n} / \mathrm{a}$ & 1 & $\mathrm{n} / \mathrm{a}$ \\
\hline Pulmonary/Cardiac & $\mathrm{n} / \mathrm{a}$ & $\mathrm{n} / \mathrm{a}$ & 2 & 0 & $\mathrm{n} / \mathrm{a}$ & 0 & $\mathrm{n} / \mathrm{a}$ & $\mathrm{n} / \mathrm{a}$ & $\mathrm{n} / \mathrm{a}$ \\
\hline MOF (Perionitis) & $\mathrm{n} / \mathrm{a}$ & $\mathrm{n} / \mathrm{a}$ & $\mathrm{n} / \mathrm{a}$ & 0 & $\mathrm{n} / \mathrm{a}$ & 0 & $\mathrm{n} / \mathrm{a}$ & $\mathrm{n} / \mathrm{a}$ & $\mathrm{n} / \mathrm{a}$ \\
\hline Death & 1 & $\mathrm{n} / \mathrm{a}$ & 0 & 0 & $\mathrm{n} / \mathrm{a}$ & 0 & $\mathrm{n} / \mathrm{a}$ & $\mathrm{n} / \mathrm{a}$ & $\mathrm{n} / \mathrm{a}$ \\
\hline Other & 5 & $n / a$ & $\mathrm{n} / \mathrm{a}$ & $\mathrm{n} / \mathrm{a}$ & & $\mathrm{n} / \mathrm{a}$ & $\mathrm{n} / \mathrm{a}$ & $\mathrm{n} / \mathrm{a}$ & $\mathrm{n} / \mathrm{a}$ \\
\hline
\end{tabular}


Table 2. Cont.

\begin{tabular}{|c|c|c|c|c|c|c|c|c|c|}
\hline Article & $\begin{array}{l}\text { R. Beard } \\
\text { et al.2020 }\end{array}$ & S. Guadagni et al., 2019 & $\begin{array}{l}\text { F. Guerra et al., } \\
2018\end{array}$ & $\begin{array}{l}\text { R. Dwyer et al., } \\
2018\end{array}$ & A. Tsung et al., 2014 & $\begin{array}{l}\text { Eric C.H. Lai et al., } \\
2011\end{array}$ & $\begin{array}{l}\text { P.C. Giulianotti } \\
\text { et al., } 2011\end{array}$ & L. Casciola et al., 2011 & E. Berber et al., 2010 \\
\hline $\begin{array}{l}\text { 90-days mortality } \\
\text { rate and } \\
\text { Estimated } \\
\text { DFS/OS }\end{array}$ & $\begin{array}{l}60 \text { patients } \\
(52.2 \%) \text { had } \\
\text { recurrence of } \\
\text { their metastatic } \\
\text { colorectal } \\
\text { cancer } \\
\text { following liver } \\
\text { resection }\end{array}$ & $\begin{array}{l}\text { FU } 22.5 \text { Months, } 0 \text { cases of } \\
\text { local recurrence } 1 \text { Year } \\
\text { DFS- } 89.5 \% \text {, } 3 \text { Year } \\
\text { DFS- } 35.8 \%\end{array}$ & $\begin{array}{c}\text { FU } 19.5 \\
\text { months-9 disease } \\
\text { deaths, } 16 \text { cases } \\
\text { recurrent disease } \\
1 \text { Year DFS/OS } \\
83.5 \% / 90.4 \%, 3 \\
\text { Year DF/OS } \\
41.9 \% / 66.1 \%\end{array}$ & $\begin{array}{c}0 \\
\text { Not mentioned }\end{array}$ & $\begin{array}{c}0 \\
\text { NB: Unable to } \\
\text { extract true data of } \\
\text { CRLM from robotic } \\
\text { arm (heteroge- } \\
\text { nous data) }\end{array}$ & $\begin{array}{c}0 \\
1 \text { Year DFS/OS } \\
85 \% / 96 \%, 3 \text { Year } \\
\text { DFS/OS 47\%/67\% }\end{array}$ & $\begin{array}{c}0 \\
\text { NB: Unable to } \\
\text { extract true data of } \\
\text { CRLM from robotic } \\
\text { arm (heteroge- } \\
\text { nous data) }\end{array}$ & $\begin{array}{c}\text { FU } 25.1 \pm 11.7 \text { months, } \\
\text { two deaths-reoccurence } \\
\text { of disease } \\
\text { NB: Unable to extract } \\
\text { true data of CRLM from } \\
\text { robotic arm } \\
\text { (heterogenous data) }\end{array}$ & $\begin{array}{c}0 \\
\text { Not mentioned }\end{array}$ \\
\hline $\begin{array}{l}\text { Cost (might not be } \\
\text { directly stated) }\end{array}$ & $\begin{array}{l}\text { accurate data on } \\
\text { cost that would } \\
\text { allow for a } \\
\text { comparison } \\
\text { between the } \\
\text { RLR and LLR } \\
\text { groups are not } \\
\text { available. }\end{array}$ & $\begin{array}{l}\text { In our experience, RAS for } \\
\text { CRLM surgical treatment } \\
\text { was feasible and played a } \\
\text { positive role even in patients } \\
\text { with multiple metastases } \\
\text { and previous or } \\
\text { synchronous surgery. RAS } \\
\text { seemed to be oncologically } \\
\text { effective in this setting, as no } \\
\text { patients experienced local } \\
\text { relapse in the treated area. }\end{array}$ & $\begin{array}{l}\text { Robotic surgery as } \\
\text { a valid option to } \\
\text { resect CRLMs } \\
\text { competently in } \\
\text { terms of both } \\
\text { surgical and } \\
\text { oncological } \\
\text { outcomes. }\end{array}$ & $\begin{array}{c}\text { All were } \\
\text { performed } \\
\text { without the need } \\
\text { for conversion, } \\
\text { thus supporting } \\
\text { efficacy. The role } \\
\text { of robotic } \\
\text { surgery in the } \\
\text { management }\end{array}$ & $\begin{array}{l}\text { Although a greater } \\
\text { proportion of robotic } \\
\text { cases were } \\
\text { completed in a } \\
\text { totally minimally } \\
\text { invasive manner, } \\
\text { there were no } \\
\text { significant benefits } \\
\text { over laparoscopic } \\
\text { techniques in } \\
\text { operative outcomes. }\end{array}$ & $\begin{array}{l}\text { The main drawback } \\
\text { of advanced robotic } \\
\text { surgery is the } \\
\text { associated cost. At } \\
\text { the current stage of } \\
\text { development, the } \\
\text { benefits of } \\
\text { robot-assisted } \\
\text { surgery in liver } \\
\text { surgery have not yet } \\
\text { been defined. }\end{array}$ & $\begin{array}{c}\text { This preliminary } \\
\text { experience shows } \\
\text { that robotic surgery } \\
\text { can be used safely } \\
\text { for liver resections } \\
\text { with a limited } \\
\text { conversion rate, } \\
\text { blood loss, and } \\
\text { postoperative } \\
\text { morbidity. }\end{array}$ & $\begin{array}{l}\text { Finally, results of this } \\
\text { series show that } \\
\text { robot-assisted LHR is safe } \\
\text { and feasible and provide } \\
\text { considerable evidence that } \\
\text { robotics could overcome } \\
\text { limitations of traditional } \\
\text { LHR associated with } \\
\text { tumor location, } \\
\text { supporting robot use only } \\
\text { for complex resections. }\end{array}$ & $\begin{array}{l}\text { The additional cost } \\
\text { incurred by the robot is } \\
\text { a concern when } \\
\text { justifying its use. } \\
\text { Although a case-by-case } \\
\text { cost analysis was not } \\
\text { done, the robotic } \\
\text { instrumentation in } \\
\text { general adds } \$ 500 \text { per } \\
\text { case to the laparoscopic } \\
\text { equipment cost. }\end{array}$ \\
\hline
\end{tabular}


Pre-operative: The average age in the LLR was 65 years compared to 63 years in the RLR. Within the LLR group, the average tumour size excised was smaller at 32.1 $\mathrm{mm}$ compared to the RLR group of $33.8 \mathrm{~mm}$. Preoperative systemic chemotherapy was administered in all of the laparoscopic studies, except one. Major hepatectomies were defined as resection of three or more segments and synchronous metastases were defined as cases in which patients received a diagnosis of CRLM at the same time or within 6 months of the diagnosis of the primary tumour.

Intra-Operative: The operative time was reported in all of the included studies. The data show that the operative time was longer in the RLR group compared with the LLR group. The average operative time in the LLR is $193 \mathrm{~min}$, CI of $95 \%$ (147.4 $\mathrm{min}$ to 238.6 $\mathrm{min}$ ) compared to RLR $257 \mathrm{~min}, \mathrm{CI}$ of $95 \%$ (201.5 $\mathrm{min}$ to $313.8 \mathrm{~min}$ ) with a $p$-value $<0.0001$. Estimated blood loss was lower in the RLR group $(210 \mathrm{~mL})$ compared with the LLR group $(246 \mathrm{~mL})$. The need for conversion was similar between individual studies and the majority of the studies did not directly comment on the use of the Pringle Manoeuvre.

Post-Operative: Oncological outcomes were mentioned in 16 studies (9 Laparoscopic, 7 Robotic), concluding high rates of successful R0 margins, independent of the modality of surgery. A total of 1035 from 1090 (95\%) patients had an R0 resection margin within the LLR group compared to 82 from 86 (95\%) patients that had an R0 resection margin within the RLR group. However, the sample sizes leading to the conclusions are significantly different, 1090 (LLR group) vs. 86 (RLR group). This may produce inconclusive results, as the statistical power to detect a difference would be low. It is clear more patients are required within the RLR group to form a robust statement. From an oncologic standpoint, local and systemic recurrence rate, recurrence site and 1- and 3-year overall and disease-free survival (DFS) were analysed as shown in Tables 1 and 2.

Cost-Effectiveness Comparison: Overall the length of hospital stay (LOS) was shorter in the RLR group at 5.9 days compared to the LLR group of 6.6 days. Within the RLR group, two studies [8,9] commented on 14 cases as grade 1-2 of the clavien-dindo classification (CDC). The LLR group had two studies $[10,11]$ that commented on 18 cases as grade 1-2 CDC. The RLR group had 4 cases that returned to theatre within 30 days post-operatively (ERCP stenting for bile leak). Within, the LLR group, 22 cases returned to theatre within 30 days post-operatively. Due to the heterogeneity of the data, the majority of studies did not directly comment on the percentages of patients re-operated in each group. The most common complication in both groups was post-operative bleeding. Overall the RLR group had a greater number of complications per case (32\%) compared to the LLR group (22.9\%).

\section{Discussion}

Surgical resection is the only curative method for CRLM and an R0 resection margin is the ultimate goal. The use of minimally invasive surgery (MIS) has recently been embraced within resectional HPB surgery. Due to the complexity of hepatic resection, there are key concerns that have to be overcome, such as inadequate margins, tumour seeding, missing small metastasis, air embolism and poor oncological outcomes. This review included reports of 1194 patients who underwent laparoscopic liver resections compared to 146 robotic liver resections for CRLMs. From the statistical findings, within the RLR group, larger tumour size was excised with a longer operative time, whereas the estimated blood loss was less compared to the LLR group. High rates of successful R0 margins, independent of the modality of surgery were noted. The mean LOS was shorter in the RLR group compared to the LLR group. The number of complications per case was greater in the RLR group compared to the LLR group. However, neither result was shown to be statistically significant.

One study on short and long-term outcomes of laparoscopic hepatectomy for colorectal liver metastases in the elderly was published by Yue et al., 2018. LLR were performed in 241 consecutive patients over the age of 60 years old with CRLMs. Based on age, they were divided into subgroups. The elderly group (70 years old and above) had 78 patients with a higher American Society of Anaesthesiologist (ASA) score [12]. There was no significant 
difference between groups in regards to operative time, blood loss and postoperative complications and they had equal oncological outcomes. Therefore laparoscopic surgery was shown to be beneficial in elderly patients with CRLMs with good short and long-term survival results. The OSLO-COMET randomised controlled trial conducted by Fretland et al., 2018 was the first of its kind to compare LLR to OLR (Open Liver Resection) for CRLMs. A single-centre, double-blinded study randomised 280 patients into two groups, 133 (LLR) and 147 (OLR). The primary outcome was postoperative complications within 30 days, which was $19 \%$ in the LLR compared to high morbidity of $31 \%$ in the OLR [6]. The operative time and blood loss were similar in both groups; however, the length of stay (LOS) was shorter in the LLR. Interestingly there was no difference in rates of R0 resection or positive margins within the groups and the cost of LLR was higher. A metaanalysis of 610 patients directly comparing laparoscopic versus open liver resection for metastatic colorectal cancer was conducted by Schiffman et al., 2014. LLR were performed in 242 patients compared to 368 patients who underwent OLR [13]. There was no difference in terms of age, sex, tumour size or number of metastasis in either group. The major findings in this study were decreased estimated blood loss, need for transfusion, LOS and overall complication rates in LLR compared to OLR. In conclusion, it was found that patients were able to tolerate laparoscopic surgery with overall reduced morbidity and good oncological outcome. However, Schiffman stated that patients with multifocal, bilobar tumours, tumours invading the inferior vena cava or confluence junction of portal vein would not be ideal candidates for Minimum Invasive Surgery (MIS). Ferretti et al., 2015 designed a multicentre international study of case series performing simultaneous laparoscopic resection of the primary colorectal cancer and synchronous CRLMs. A total of 142 cases were performed and the median number of liver lesions was $1(1-9)$ with an average operative time of 360 (120-690) $\mathrm{min}$ [10]. The most important finding was the global morbidity of $31 \%$, lower than comparable open studies. Abu Hilal et al., 2012 conducted a large single-centre study, of 133 laparoscopic liver resections within the UK. A total of 83 cases were CRLMs related and their study demonstrates that the laparoscopic approach is feasible and safe, even when more extreme repeated hepatectomies for recurrent CRLM or 2-stage laparoscopic liver resections for bilobar CRLM are required [14]. An R0 resection margin was obtained in 112 of 116 resections (97\%).

It is noted that there are limitations to laparoscopic liver surgery, such as loss of depth perception (stereopsis) in a two-dimensional field, fulcrum effect against ports, a limited degree of movement required for meticulous steps leading to reduced surgical accuracy and tremor intensification. Robotic surgery was developed to overcome many of these limitations. Fundamental features such as instruments that provide better ergonomics with a 7 degree of freedom for movement and tremor filtering, three-dimensional imaging will display deformation of deeper structures and more precise anatomy. Casciola et al., 2011 described the benefits of robotic liver surgery, particularly in resecting lesions in the posterior-superior segments (VII and VIII) as they required curved transections, hepatocaval dissection and bilioenteric reconstruction, which are very challenging with laparoscopy [15]. The surgeon is able to control the camera and instruments simultaneously, eliminating inappropriate camera control or retraction. The presence of computerised console can allow image-guided surgery that is becoming attractive in hepatobiliary surgery [16]. Pesi et al., 2019 analysed surgical and oncological outcomes of a series of 51 patients who received ultrasound-guided robotic liver resections (RLRs) for both primary and secondary malignancies. Their results showed that robotic liver surgery is effective with acceptable uncompromised long-term oncological outcomes [17]. Fruscione et al., 2019 compared robotic-assisted (57 cases) versus laparoscopic (116 cases) major liver resections from a single centre. They concluded that patients who underwent robotic surgery had a lower rate of postoperative ICU admission and lower re-admission rate within 90 days compared to the laparoscopic group [18]. This study has several limitations. The retrospective nature of the study incorporates selection bias. Two surgeons with no evidence of case matching performed all the cases. Purposing that a major hepatectomy 
and technically challenging cases (high risk of bleeding or achieving R0 resection) should be performed robotically. Especially as robotic surgery has superior ergonomics and can overcome surgical fatigue delivered by prolonged laparoscopy.

Guerra et al., 2018 published a multi-centre study of 59 patients receiving ultrasoundguided robotic resection for CRLMs. A total of 82 liver resections were performed with a median operative time of $210 \mathrm{~min}$ and an estimated blood loss of $200 \mathrm{~mL}$ [8]. Overall 7 operations were converted to open surgery, mainly due to uncontrolled bleeding during parenchymal transection. The mean LOS was 6 days and R0 resection was achieved for $92 \%$ of lesions. This study illustrates that robotic surgery is effective in resecting CRLMs with similar oncological outcomes to laparoscopic or open surgery. Dwyer et al., 2018 presented a series of synchronous robotic surgery for CRLMs. The retrospective study consisted of 6 patients who underwent colonic resections with an average of 2.25 liver segmentectomy. In all cases, liver surgery was performed first. A mean operative time of $401 \mathrm{~min}$ was found, with an estimated blood loss of $316 \mathrm{~mL}$, no conversion to open, LOS of 4.5 days and no 30-day mortality [19]. This small case series illustrates that one-stage synchronous robotic surgery is safe and feasible in well-selected patients. Tsung et al., 2014 published the largest series comparing robotic to laparoscopic liver resections. A total of 57 robotic liver resections were compared to 114 laparoscopic cases. In regards to CRLMs, 21 patients had RLR compared to 36 LLR. There were no significant differences between the operative and postoperative outcomes of robotic and laparoscopic groups. Both had similar oncological margins. However, the robotic group had a significantly greater overall room time (342 vs. $261.5 \mathrm{~min}, p<0.001$ ) and Operating time (253 vs. $198.5 \mathrm{~min}, p=0.001$ ) [20]. The likely reason being the additional time required for docking the robot, exchange and repositioning of instruments. A greater number of complex major hepatectomies were performed robotically with a total minimally invasive technique, which would be favorable to patients and many surgeons.

Major hepatectomy or resections in posterior-superior segments have been described to be difficult even in laparoscopic procedures, and these cases theoretically may benefit from robotic surgery. Within our results, Montalti et al., exclusively compare robot-assisted to laparoscopic parenchymal-preserving liver posterior superior resections for lesions located in segments 7, 8, 4a, and 1 . Thirty-six patients who underwent RLR were matched with 72 patients undergoing LLR. There were no significant differences in postoperative outcomes as measured by blood loss, hospital stay, R0 negative margin rate, and mortality [21]. Ziogas et al., performed a meta-analysis specifically addressing the comparison between major liver resections performed laparoscopically and robotically [22]. Seven retrospective cohort studies comparing LLR $(n=300)$ versus RLR $(n=225)$ were identified. There was no significant difference observed between the overall complication rates between the two groups. There was no significant difference observed for blood loss, operative time and length of stay. Rahimli et al. compared their robotic and laparoscopic liver surgery for colorectal liver metastases results from a single study centre in Germany. The proportion of major resections was higher in the robotic group with five patients (3 left hemi-hepatectomy, 1 right hemi-hepatectomy, $>3$ segmental resections). The laparoscopic major resection group consisted of three patients ( 1 right hemi-hepatectomy, $>3$ segmental resections, 3 segmental resections). Direct comparison of major resections showed robotic surgery to be longer (412 $\mathrm{min}$ in RLR vs. $363.6 \mathrm{~min}$ in LLR), less peri-operative blood loss ( $600 \mathrm{~mL}$ in RLR vs. $762.5 \mathrm{ml}$ in LLR) and longer length of stay (12.4 days in RLR vs. 11.5 days in LLR) [23]. There was a trend towards higher R0 resections in the robotic group; this may be related to more precise control of parenchymal dissection.

Daskalak et al., 2017 assessed the clinical outcomes and economic impact of robotic and open liver surgery in a single centre. Within the robotic group, there were 68 robotic cases ( 29 major, 39 minor) compared to the open group of 55 cases ( 24 major, 31 minor). An independent company performed the financial analysis comparing six categories of cost (anaesthesiology, operating room, ICU, nursing, pharmacy and re-admission). On average, there was no difference in anaesthetic costs between the two surgery types. It was 
noted, however, the mean estimated blood loss, complication rates and overall morbidity were considerably less in the robotic group. The length of stay in the intensive care unit (ICU) was shorter for patients who underwent a robotic procedure (2.1 versus 3.3 days; $p=0.004$ ) [24]. The average total cost, including readmissions, was less for the robotic group compared to the open group. Their study illustrated that using the robot for complex cases would lead to an overall reduction in complications, directly impacting on a shorter length of hospital stay and re-admission costs. With time this would become a real cost-benefit, even if the initial intraoperative costs are higher. Bearing in mind this is all supposition and unproven. The key message is remembering the early financial impact of laparoscopic liver resections had when compared to open surgery. Initially, there is higher intraoperative cost and longer operative times, however, a clear benefit in a shorter length of hospital stay and morbidity leading to a reduction in overall hospital costs [25]. Fretland et al., 2018 estimated in a 4-month health care perspective that laparoscopy had a 95,000 USD threshold and was cost-effective compared to open surgery [6]. However, Berber et al., 2010 stated the additional cost gained by robotic instrumentation adds 500 USD per case to the laparoscopic equipment cost [26]. The additional operative time in RLRs compared to LLRs was ascribed to unfamiliarity with the new technology, setup and learning curve.

Several limitations of our study should be mentioned. Firstly, the studies included a small number of patients in the robotic group compared to the laparoscopic group. All data regarding patient demographics, tumour characteristics, intraoperative variables, and postoperative outcomes were retrospectively collected from medical records incorporates selection bias. RLR group studies did not directly state whether docking time was included in the operative time. The heterogeneity of data directly weakens the significance of our statistical results of weighing advantages and disadvantages. Many studies had insufficient information to allow exact criteria matching.

\section{Conclusions}

Despite the higher operative cost, RLRs do not result in statistically better treatment outcomes, with the exception of lower estimated blood loss and excision of larger CRLMs. Operative time and total complication rate are significantly more favourable with LLRs. Our study has shown that robotic liver surgery is safe and feasible in well-selected patients. Major advantages over laparoscopy are 3D imaging, better ergonomics leading to precise operative dexterity and improved surgical comfort. However, the available evidence on robotic liver surgery is still limited and definitive conclusions on the actual role of robotics cannot be drawn, especially long-term oncological and cost benefits. Furthermore, a welldesigned, randomised control study is required to assess the actual advantages of robotic over laparoscopic liver surgery for CRLMs.

Author Contributions: Conceptualisation, N.M. and H.A.; methodology, N.M., H.A. and B.A.A.; validation, N.M., H.A., T.C. and B.A.A.; formal analysis, N.M., H.A. and B.A.A.; writing-original draft preparation, N.M., H.A. and T.C.; writing-review and editing, N.M. and H.A.; supervision, R.L., T.A.R. and A.E.F. All authors have read and agreed to the published version of the manuscript.

Funding: This research received no external funding.

Institutional Review Board Statement: Not applicable.

Informed Consent Statement: Not applicable.

Conflicts of Interest: The authors declare no conflict of interest.

\section{References}

1. Salvatore, L.; Aprile, G.; Arnoldi, E.; Aschele, C.; Carnaghi, C.; Cosimelli, M.; Maiello, E.; Normanno, N.; Sciallero, S.; Valvo, F.; et al. Management of metastatic colorectal cancer patients: Guidelines of the Italian Medical Oncology Association (AIOM). ESMO Open 2017, 2, e000147. [CrossRef] [PubMed]

2. Cancer Research UK. Available online: https://www.cancerresearchuk.org/health-professional/cancer\%20statistics/statisticsby-cancer-type/bowel-cancer\#heading-Zero (accessed on 9 February 2020). 
3. Weiss, L.; Grundmann, E.; Torhorst, J.; Hartveit, F.; Moberg, I.; Eder, M.; Fenoglio-Preiser, C.M.; Napier, J.; Horne, C.H.W.; Lopez, M.J.; et al. Haematogenous metastatic patterns in colonic carcinoma: An analysis of 1541 necropsies. J. Pathol. 1986, 150, 195-203. [CrossRef] [PubMed]

4. Jongen, J.M. Disease Progression and Therapy-Induced Changes in Metastatic Colorectal Cancer. Ph.D. Thesis, Utrecht University, Utrecht, The Netherlands, 2019.

5. Wayand, W.; Woisetschläger, R. Laparoscopic resection of liver metastasis. Chir. Z. Geb. Oper. Medizen 1993, 64, $195-197$.

6. Fretland, Å.A.; Dagenborg, V.J.; Bjørnelv, G.M.W.; Kazaryan, A.M.; Kristiansen, R.; Fagerland, M.W.; Hausken, J.; Tønnessen, T.I.; Abildgaard, A.; Barkhatov, L.; et al. Laparoscopic Versus Open Resection for Colorectal Liver Metastases: The OSLO-COMET Randomized Controlled Trial. Ann. Surg. 2018, 267, 199-207. [CrossRef]

7. Royal College of Pathology. Standards and Datasets for Reporting Cancers. Available online: http://www.rcpath.org/index (accessed on 4 April 2020).

8. Guerra, F.; Guadagni, S.; Pesi, B.; Furbetta, N.; Di Franco, G.; Palmeri, M.; Annecchiarico, M.; Eugeni, E.; Coratti, A.; Patriti, A.; et al. Outcomes of robotic liver resections for colorectal liver metastases. A multi-institutional analysis of minimally invasive ultrasound-guided robotic surgery. Surg. Oncol. 2019, 28, 14-18. [CrossRef]

9. Guadagni, S.; Furbetta, N.; Di Franco, G.; Palmeri, M.; Gianardi, D.; Bianchini, M.; Guadagnucci, M.; Pollina, L.; Masi, G.; Cremolini, C.; et al. Robotic-assisted surgery for colorectal liver metastasis: A single-centre experience. J. Minim. Access Surg. 2019, 16, 160-165. [CrossRef]

10. Yue, M.; Li, S.; Yan, G.; Li, C.; Kang, Z. Short- and long-term outcomes of laparoscopic hepatectomy for colorectal liver metastases in elderly patients. Cancer Manag. Res. 2018, 10, 2581-2587. [CrossRef]

11. Schiffman, S.C.; Kim, K.H.; Tsung, A.; Marsh, J.W.; Geller, D.A. Laparoscopic versus Open Liver Resection for Metastatic Colorectal Cancer: A Metaanalysis of 610 Patients. Surgery 2015, 157, 211-222. [CrossRef]

12. Shim, J.R.; Lee, S.D.; Park, H.M.; Lee, E.C.; Park, B.; Han, S.S.; Kim, S.H.; Park, S.J. Outcomes of liver resection in patients with colorectal liver metastases by laparoscopic or open surgery. Ann. Hepatobiliary Pancreat. Surg. 2018, 22, 223-230. [CrossRef]

13. Ferretti, S.; Tranchart, H.; Buell, J.F.; Eretta, C.; Patriti, A.; Spampinato, M.G.; Huh, J.W.; Vigano, L.; Han, H.S.; Ettorre, G.M.; et al. Laparoscopic Simultaneous Resection of Colorectal Primary Tumor and Liver Metastases: Results of a Multicenter International Study. World J. Surg. 2015, 39, 2052-2060. [CrossRef]

14. Hilal, M.A.; Di Fabio, F.; Salameh, M.A.; Pearce, N.W. Oncological efficiency analysis of laparoscopic liver resection for primary and metastatic cancer: A singlecenter UK experience. Arch. Surg. 2012, 147, 42-48. [CrossRef] [PubMed]

15. Casciola, L.; Patriti, A.; Ceccarelli, G.; Bartoli, A.; Ceribelli, C.; Spaziani, A. Robot-assisted parenchymal-sparing liver surgery including lesions located in the posterosuperior segments. Surg. Endosc. 2011, 25, 3815-3824. [CrossRef]

16. Kingham, T.P.; Scherer, M.A.; Neese, B.W.; Clements, L.W.; Stefansic, J.D.; Jarnagin, W.R. Image-guided liver surgery: Intraoperative projection of computed tomography images utilizing tracked ultrasound. HPB 2012, 14, 594-603. [CrossRef] [PubMed]

17. Pesi, B.; Moraldi, L.; Guerra, F.; Tofani, F.; Nerini, A.; Annecchiarico, M.; Coratti, A. Surgical and oncological outcomes after ultrasound-guided robotic liver resections for malignant tumor. Analysis of a prospective database. Int. J. Med Robot. Comput. Assist. Surg. 2019, 15, e2002. [CrossRef] [PubMed]

18. Fruscione, M.; Pickens, R.; Baker, E.H.; Cochran, A.; Khan, A.; Ocuin, L.; Iannitti, D.A.; Vrochides, D.; Martinie, J.B. Roboticassisted versus laparoscopic major liver resection: Analysis of outcomes from a single center. HPB 2019, 21, 906-911. [CrossRef]

19. Dwyer, R.H.; Scheidt, M.J.; Marshall, J.S.; Tsoraides, S.S. Safety and efficacy of synchronous robotic surgery for colorectal cancer with liver metastases. J. Robot Surg. 2018, 12, 603-606. [CrossRef] [PubMed]

20. Tsung, A.; Geller, D.A.; Sukato, D.C.; Sabbaghian, S.; Tohme, S.; Steel, J.; Marsh, W.; Reddy, S.K.; Bartlett, D.L. Robotic Versus Laparoscopic Hepatectomy: A matched comparison. Ann. Surg. 2014, 259, 549-555. [CrossRef] [PubMed]

21. Montalti, R.; Scuderi, V.; Patriti, A.; Vivarelli, M.; Troisi, R.I. Robotic versus laparoscopic resections of posterosuperior segments of the liver: A propensity score-matched comparison. Surg. Endosc. 2016, 30, 1004-1103. [CrossRef]

22. Ziogas, I.A.; Giannis, D.; Esagian, S.M.; Economopoulos, K.P.; Tohme, S.; Geller, D.A. Laparoscopic versus robotic major hepatectomy: A systematic review and meta-analysis. Surg. Endosc. 2021, 35, 524-535. [CrossRef]

23. Rahimli, M.; Perrakis, A.; Schellerer, V.; Gumbs, A.; Lorenz, E.; Franz, M.; Arend, J.; Negrini, V.R.; Croner, R.S. Robotic and laparoscopic liver surgery for colorectal liver metastases: An experience from a German Academic Center. World J. Surg. Onc. 2020, 18, 333. [CrossRef]

24. Daskalaki, D.; Gonzalez-Heredia, R.; Brown, M.; Bianco, F.M.; Tzvetanov, I.; Davis, M.; Kim, J.; Benedetti, E.; Giulianotti, P.C. Financial Impact of the Robotic Approach in Liver Surgery: A Comparative Study of Clinical Outcomes and Costs Between the Robotic and Open Technique in a Single Institution. J. Laparoendosc. Adv. Surg. Tech. 2017, 27, 375-382. [CrossRef] [PubMed]

25. Vanounou, T.; Steel, J.L.; Nguyen, K.T.; Tsung, A.; Marsh, J.W.; Geller, D.A.; Gamblin, T.C. Comparing the clinical and economic impact of laparoscopic versus open liver resection. Ann. Surg. Oncol. 2010, 17, 998-1009. [CrossRef] [PubMed]

26. Berber, E.; Akyildiz, H.Y.; Aucejo, F.; Gunasekaran, G.; Chalikonda, S.; Fung, J. Robotic versus laparoscopic resection of liver tumours. HPB 2010, 12, 583-586. [CrossRef] [PubMed] 